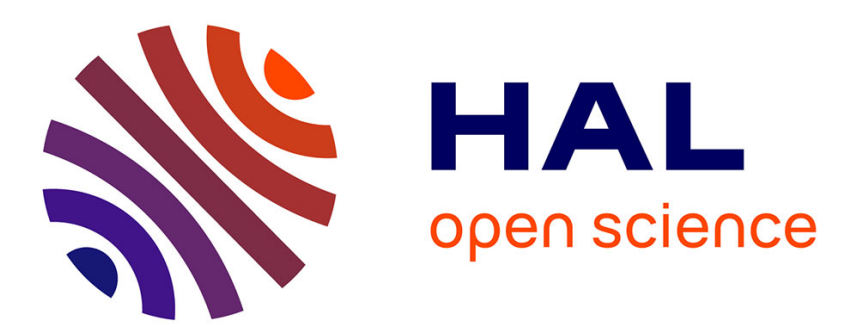

\title{
A New Method for Occupancy Grid Maps Merging: Application to Multi-vehicle Cooperative Local Mapping and Moving Object Detection in Outdoor Environment
}

Hao Li, Fawzi Nashashibi

\section{- To cite this version:}

Hao Li, Fawzi Nashashibi. A New Method for Occupancy Grid Maps Merging: Application to Multi-vehicle Cooperative Local Mapping and Moving Object Detection in Outdoor Environment. ICARCV 2012 - 12th International Conference on Control, Automation, Robotics and Vision, Dec 2012, Guangzhou, China. hal-00766777

\section{HAL Id: hal-00766777 \\ https://hal.inria.fr/hal-00766777}

Submitted on 18 Dec 2012

HAL is a multi-disciplinary open access archive for the deposit and dissemination of scientific research documents, whether they are published or not. The documents may come from teaching and research institutions in France or abroad, or from public or private research centers.
L'archive ouverte pluridisciplinaire HAL, est destinée au dépôt et à la diffusion de documents scientifiques de niveau recherche, publiés ou non, émanant des établissements d'enseignement et de recherche français ou étrangers, des laboratoires publics ou privés. 


\section{A New Method for Occupancy Grid Maps Merging: Application to Multi-vehicle Cooperative Local Mapping and Moving Object Detection in Outdoor Environment}

\author{
Hao LI \\ IMARA team, INRIA \\ Le Chesnay, France \\ hao.li@inria.fr
}

\author{
Fawzi NASHASHIBI \\ IMARA team, INRIA \\ Le Chesnay, France \\ fawzi.nashashibi@inria.fr
}

\begin{abstract}
Autonomous mapping, especially in the form of SLAM (Simultaneous Localization And Mapping), has long since been used for many indoor robotic applications and is also useful in outdoor intelligent vehicle applications such as object detection. Most existing research works on environment mapping and object detection in outdoor applications have been dedicated to single vehicle system. On the other hand, multi-vehicle cooperative perception based on inter-vehicle data sharing can bring considerable benefits in many scenarios that are challenging for a single vehicle system. In this paper, a new method for occupancy grid maps merging is proposed: an objective function based on occupancy likelihood is introduced to measure the consistency degree of maps alignment; genetic algorithm implemented in a dynamic scheme is adopted to optimize the objective function. A scheme of multi-vehicle cooperative local mapping and moving object detection using the proposed occupancy grid maps merging method is also introduced. Real-data tests are given to demonstrate the effectiveness of the introduced method.
\end{abstract}

Keywords-occupancy grid map, SLAM, cooperative perception, moving object detection

\section{INTRODUCTION}

Autonomous mapping has long since been a fundamental task for mobile robots operating in an unknown environment [1]. Since the robot pose is not known a priori in many applications, the mapping process is usually juxtaposed with the localization process, forming a process called Simultaneous Localization And Mapping (SLAM) [2] [3]. Originally, SLAM was rooted in indoor environment applications, where the purpose of SLAM is to establish a consistent spatial representation for the global environment and localize the robot in this global environment representation. For outdoor environment applications where GPS (Global Positioning System) measurements can be available, SLAM gradually loses its importance on global mapping and global localization as originally in indoor environment applications. Nevertheless, SLAM methods can still be employed to improve odometerbased local localization result and build accurate and consistent local maps for object detection [4] [5].
Most existing research works on environment mapping and object detection in outdoor environment applications have been dedicated to single vehicle system (the term "vehicle" and "robot" are used interchangeably in this paper). On the other hand, multi-vehicle cooperative perception based on intervehicle data sharing can bring considerable benefits in many scenarios that are challenging for a single vehicle system. Take vehicle overtaking scenario as an example, see Fig.1; this scenario is challenging and potentially dangerous, because the view of the overtaking vehicle is occluded by the overtaken vehicle. What might happen to the overtaking vehicle if a careless pedestrian is rushing across the road in front of the overtaken vehicle? For safety reason, the overtaking vehicle always wants to know what exist in the occluded area; unfortunately, it can not have any inference on the occluded area.

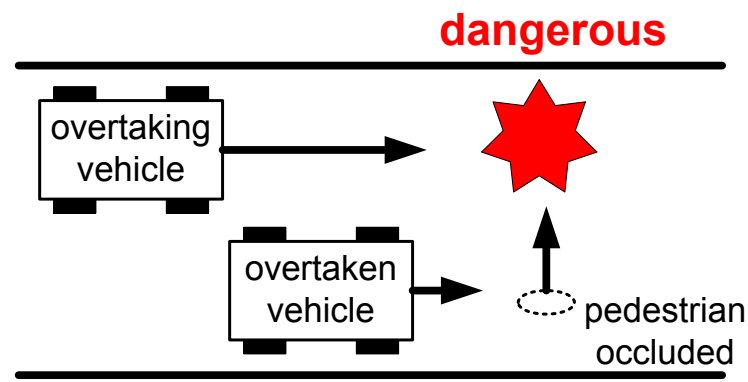

Fig 1 Potentially dangerous overtaking scenario

With the help of inter-vehicle communication [6], the overtaken vehicle can share its perception results with the overtaking vehicle so that the overtaking vehicle can indirect 'perceive' the occluded environment. More specifically, the term perception here implies certain local map representation around the vehicle. Then, an essential requirement for realizing this idea of cooperative perception is to merge the local maps of the two vehicles into a consistent local map. 
If each vehicle is precisely localized in the same global reference, then the local maps from different vehicles can be related to each other using the precise localization results and local maps merging can be effectively solved. However, a vehicle is usually not precisely localized in reality; for a vehicle equipped with low-cost GPS, the global localization error can be as large as ten meters in the position component. Local maps merging at the existence of large vehicle localization error is not trivial.

A typical practice for maps merging is based on estimation of inter-vehicle relative pose [7] [8] [9] [10], which is not easy to tackle in outdoor traffic environment: first, reliable vehicle detection and recognition are still a challenging problem that deserves further research works. Second, data association is also a challenging problem, especially when vehicle localization accuracy is low. Special patterns might be designed to facilitate vehicle detection as well as data association. However, thousands of vehicles exist in traffic environment. The task of designing proper patterns to distinguish such huge number of vehicle systems is not trivial; besides, occlusions might cause miss detection and false detection of these patterns. Even if vehicle detection and data association are performed correctly, the detection result usually corresponds to partial contour of the detected vehicle (sometimes of irregular shape), which makes it difficult to extract accurate geometric information of the detected vehicle.

Another practice for maps merging is to merge the maps directly [11], completely ignoring the issue of inter-vehicle relative pose estimation. We focus on merging occupancy grid maps [12] [13] instead of feature-based maps [14], for the general environment representation capability of occupancy grid maps. Moreover, as in [11], we want to avoid any preliminary procedure of feature extraction and focus on directly identifying the alignment of the maps to-be-merged.

In this paper, a new method for occupancy grid maps merging is proposed (this method has been used as a component in our previous works and has been briefly mentioned in [17]; here the method is presented with more details): an objective function based on occupancy likelihood is introduced to measure the consistency degree of maps alignment; genetic algorithm implemented in a dynamic scheme is adopted to optimize the objective function. Then a scheme of multi-vehicle cooperative local mapping and moving object detection is described, which utilizes the occupancy grid maps merging method. The paper is arranged as follows: single vehicle local SLAM based on occupancy grids and vehicle global localization based on GPS are briefly reviewed in Section 2. The proposed occupancy grid maps merging method is introduced in Section 3. The scheme of multi-vehicle cooperative moving object detection is presented in Section 4. Real-data experiments are given in Section 5, followed by a conclusion in Section 6.

\section{Single VEHIClE LOCALIZATION AND MAPPING}

\section{A. Single Vehicle Local SLAM based on Occupancy Grid}

Occupancy grid based map representation is used for its ability to represent general unstructured outdoor environment. The occupancy grid is a two-dimensional lattice of rectangular cells and each cell is associated with a real value in the unit interval $[0,1]$. The cell value represents the degree of the cell being occupied or free. The cell value 0.5 represents the cell being in unknown state, neither occupied nor free. For cell value larger than 0.5 , the larger the cell value is, the more likely the cell is occupied. For cell value smaller than 0.5 , the smaller the cell value is, the more likely the cell is free.

Here, we adopt the incremental maximum likelihood SLAM method in [5], considering its computational efficiency and its insensitiveness to dynamic entities; this method is briefly reviewed. Generally, let $\mathbf{x}$ denotes vehicle local pose in SLAM, M denotes updated map, $\mathbf{u}$ denotes odometer data, $\mathbf{z}$ denotes range data. Let subscript $t$ denotes the time index. The incremental maximum likelihood SLAM is a repeated process of executing procedures (1) and (2):

$$
\begin{gathered}
\hat{\mathbf{x}}_{t}=\underset{x_{t}}{\arg \max }\left\{P\left(\mathbf{z}_{t} \mid \mathbf{x}_{t}, \hat{\mathbf{M}}_{t-1}\right) \cdot P\left(\mathbf{x}_{t} \mid \hat{\mathbf{x}}_{t-1}, \mathbf{u}_{t}\right)\right\} \\
\hat{\mathbf{M}}_{t}=\hat{\mathbf{M}}_{t-1} \cup\left\{\hat{\mathbf{x}}_{t}, \mathbf{z}_{t}\right\}
\end{gathered}
$$

The procedure (1) is to search the optimal $x_{t}$ which maximizes the marginal likelihood of the $t$-th pose and perception relative to the ( $t-1)$-th pose and map; the grid-based maximum likelihood scan matching method in [5] is used for the searching. The procedure (2) is to generate a new ( $t$-th) map from the old ((t-1)-th) map based on the estimated $t$-th pose and the $t$-th perceptive data. Detailed implementation is referred to [5]. An example of two local maps built by different vehicles is shown in the top two sub-figures of Fig.2.

\section{B. Single Vehicle Global Localization based on GPS}

GPS can provide direct and error-bounded global position measurement; the measurement error level depends on the GPS quality as well as the environment where the GPS operates. In comparatively open area, a RTK-GPS can achieve centimeterlevel positioning accuracy; in contrast, the positioning error of a low-cost GPS can be ten meters. Normally, a GPS outputs measurements at a low frequency and does not provide direct measurement on vehicle orientation, a filtering process is usually performed to estimate full state of vehicle pose (position and orientation). In the presented works, EKF (Extended Kalman Filter) is used for the filtering process, as follows:

\section{1) Global pose evolution}

The evolution of vehicle pose can be modeled according to kinematic bicycle model (denoted generally as function $\mathbf{F}$ ):

$$
\begin{gathered}
\mathbf{X}_{t}=\mathbf{F}\left(\mathbf{X}_{t-1}, \mathbf{u}_{t}\right) \\
\boldsymbol{\Sigma}_{\mathbf{X}_{t}}=\mathbf{F}_{\mathbf{X}} \boldsymbol{\Sigma}_{\mathbf{X}_{t-1}} \mathbf{F}_{\mathbf{X}}{ }^{T}+\mathbf{F}_{\mathbf{u}} \boldsymbol{\Sigma}_{\mathbf{u}} \mathbf{F}_{\mathbf{u}}{ }^{T}
\end{gathered}
$$

The $\mathbf{X}$ denotes vehicle global pose (different from the vehicle local pose $\mathbf{x}$ ); $\mathbf{u}$ denotes vehicle motion variables, which can be odometer data. In order to reduce odometer error, the local SLAM described in the previous sub-section is used to correct odometer data and the corrected odometer data are used as ' $\mathbf{u}$ ' 
in (3a). The $\mathbf{F}_{\mathbf{X}}$ and $\mathbf{F}_{\mathbf{u}}$ in (3b) respectively denote the Jacobian matrices of the function $\mathbf{F}$ with respect to $\mathbf{X}$ and $\mathbf{u}$.

\section{2) Global pose update}

Let the GPS measurement be denoted as $\mathbf{Z}_{\mathrm{gps}}=\left(x_{\mathrm{gps}}, y_{\mathrm{gps}}\right)$. The measurement model can be described as:

$$
\mathbf{Z}_{g p s}=\mathbf{H}_{g p s} \mathbf{X}_{t}+\mathbf{E}_{g p s}
$$

where $\mathbf{H}_{\mathrm{gps}}=\left[\begin{array}{ll}\mathbf{I}_{2 \times 2} & \mathbf{0}_{2 \times 2}\end{array}\right]$; the measurement error $\mathbf{E}_{\mathrm{gps}}$ is assumed to follow the Gaussian distribution $\mathrm{N}\left(\mathbf{0}, \boldsymbol{\Sigma}_{\mathrm{gps}}\right)$. The global pose is updated with the GPS measurement:

$$
\begin{aligned}
& \mathbf{K}=\boldsymbol{\Sigma}_{\mathbf{X}_{t}} \mathbf{H}_{g p s}{ }^{T}\left(\mathbf{H}_{g p s} \boldsymbol{\Sigma}_{\mathbf{X}_{t}} \mathbf{H}_{g p s}{ }^{T}+\boldsymbol{\Sigma}_{g p s}\right)^{-1} \\
& \mathbf{X}_{t}=\mathbf{X}_{t}+\mathbf{K}\left(\mathbf{Z}_{g p s}-\mathbf{H}_{g p s} \mathbf{X}_{t}\right) \\
& \boldsymbol{\Sigma}_{\mathbf{X}_{t}}=\left(\mathbf{I}-\mathbf{K} \mathbf{H}_{g p s}\right) \boldsymbol{\Sigma}_{\mathbf{X}_{t}}
\end{aligned}
$$

\section{OCCUPANCY GRID MAPS MERGING}

\section{A. An objective function based on occupancy likelihood}

We follow the compounding notation in [4]:

$$
\begin{aligned}
& {\left[\begin{array}{l}
x_{1} \\
y_{1} \\
\theta_{1}
\end{array}\right] \oplus\left[\begin{array}{l}
x_{2} \\
y_{2} \\
\theta_{2}
\end{array}\right] }=\left[\begin{array}{c}
x_{2} \cos \theta_{1}-y_{2} \sin \theta_{1}+x_{1} \\
x_{2} \sin \theta_{1}+y_{2} \cos \theta_{1}+y_{1} \\
\theta_{2}+\theta_{1}
\end{array}\right] \\
& {\left[\begin{array}{l}
x_{1} \\
y_{1} \\
\theta_{1}
\end{array}\right] \oplus\left[\begin{array}{l}
x_{2} \\
y_{2}
\end{array}\right]=\left[\begin{array}{l}
x_{2} \cos \theta_{1}-y_{2} \sin \theta_{1}+x_{1} \\
x_{2} \sin \theta_{1}+y_{2} \cos \theta_{1}+y_{1}
\end{array}\right] } \\
&\left.\operatorname{inv}\left(\begin{array}{l}
x \\
y \\
\theta
\end{array}\right]\right)=\left[\begin{array}{c}
-x \cos \theta-y \sin \theta \\
x \sin \theta-y \cos \theta \\
-\theta
\end{array}\right]
\end{aligned}
$$

Let $\mathbf{M}_{\mathrm{A}}$ and $\mathbf{M}_{\mathrm{B}}$ be two occupancy grid maps to-be-merged. The process of occupancy grid maps merging can be generalized as the following optimization problem: First, design an objective function $F_{c}$ in terms of two arbitrary occupancy grid maps $\mathbf{M}_{1}$ and $\mathbf{M}_{2}$, i.e. $F_{c}\left(\mathbf{M}_{1}, \mathbf{M}_{2}\right)$, which is used to measure their consistency degree. Second, search the optimal relative pose $\mathbf{p}_{\mathrm{BA}}$ that maximizes the consistency measure between $\mathbf{M}_{\mathrm{A}}$ and $\mathbf{p}_{\mathrm{BA}} \oplus \mathbf{M}_{\mathrm{B}}$, i.e.

$$
\hat{\mathbf{p}}_{\mathrm{BA}}=\underset{\mathbf{p}_{\mathrm{BA}}}{\arg \max } F_{c}\left(\mathbf{M}_{\mathrm{A}}, \mathbf{p}_{\mathrm{BA}} \oplus \mathbf{M}_{\mathrm{B}}\right)
$$

In [11], the objective function $F_{c}$ consists of a similarity term and a lock term: the similarity term which is based on a distance-map represents the overall distances between the maps to-be-merged; the lock term is a part heuristically added to counteract the over-fitting effect. This objective function in [11] has two major disadvantages: first, the parameter $\mathrm{c}_{\text {lock }}$ in the heuristically added lock term has to be tuned empirically according to concrete scenarios.
Second, this objective function is sensitive to maps inherent inconsistency i.e. maps inconsistency that still exists even if the maps to-be-merged are aligned correctly. Maps inherent inconsistency can be caused by dynamic entities which are common in outdoor environment. Maps inherent inconsistency can also be caused by the inconsistency of perception poses at different vehicles; for example, the same environment might appear noticeably different if it is scanned by laser scanners at different heights. For the objective function in [11], maps inherent inconsistency would cause drastic value change in the distance-map based similarity term and false counting of agreement and disagreement in the lock term.

Here, we use an objective function based on occupancy likelihood, similar to the idea of the occupancy grid based scan matching as introduced in [5]. Let the occupied cells with local maximum occupancy state (referred to as local maximum occupied cells) in $\mathbf{M}_{\mathrm{B}}$ be denoted as a set of two-dimensional points $\left\{\mathbf{0}_{\mathrm{B}(1)}, \mathbf{0}_{\mathrm{B}(2)}, \ldots, \mathbf{0}_{\mathrm{B}(n b)}\right\}$. Let the occupancy state of a point $\mathbf{p}$ in an occupancy-grid map $\mathbf{M}$ be denoted as $\mathbf{M}(\mathbf{p})$; then the objective function $F_{c}$ is defined as in (6):

$$
F_{c}\left(\mathbf{M}_{\mathrm{A}}, \mathbf{p}_{\mathrm{BA}} \oplus \mathbf{M}_{\mathrm{B}}\right)=\sum_{i=1}^{n b} \mathbf{p}_{\mathrm{BA}} \oplus \mathbf{o}_{\mathrm{B}(i)} \in O c c\left(\mathbf{M}_{\mathrm{A}}\right)\left\{\mathbf{M}_{\mathrm{A}}\left(\mathbf{p}_{\mathrm{BA}} \oplus \mathbf{o}_{\mathrm{B}(i)}\right)\right\}
$$

The objective function (6) computes the occupancy likelihood sum of the local maximum occupied cells of $\mathbf{M}_{\mathrm{B}}$ in $\mathbf{M}_{\mathrm{A}}$. The Occ means the set of occupied cells, which are selected by a threshold. Here, the Occ threshold is not intended to determine whether a grid cell is truly occupied or not in reality; it is only used to select grid cells that tend to be occupied or be closer to truly occupied cells. So there is fair flexibility in setting this threshold. For example, we can set the Occ threshold to be 0.6 .

This objective function only takes into account the consistent part of the maps to-be-merged; thus it is insensitive to maps inherent inconsistency. For local maps of enough size, stable and consistent objects (buildings, infrastructures etc) are usually the dominating factors, which always contribute to successful local maps merging.

\section{B. Optimization using genetic algorithm}

The initial value of $\mathbf{p}_{\mathrm{BA}}$ can be computed with GPS based vehicle global localization results, yet this initial value might be far away from the optimal maps alignment. For intelligent vehicle systems with low-accuracy GPS, the initial position error of $\mathbf{p}_{\mathrm{BA}}$ can be twenty meters; initial orientation error of $\mathbf{p}_{\mathrm{BA}}$ can also be large. Besides, the value space of the objective function (6) is normally multimodal and of irregular shape on the whole. Therefore, local optimization searching techniques such as gradient based analytical techniques tend to fail when facing such large initial estimate error.

The strategy of evolutionary genetic algorithm [15] is adopted to solve the optimization problem (5). One important motivation for using genetic algorithm is that it is independent of the objective function value space and it is ready to solve multimodal, non-differentiable, or non-continuous problems. 
Genetic algorithm is rather a methodology instead of being a list of concrete execution procedures. As an analogy to species evolution under the influence of natural selection, the fundamental spirit of genetic algorithm is to evaluate the fitness values of a group of tentative solution individuals, vary them with biologically inspired operations such as crossover and mutation, and keep those better individuals. The concrete procedures to put this spirit into practice are problem oriented and can be specially designed and modified. The concrete procedures in our implementation are as follows:

1. Initialization: randomly generate an initial population of $\mathbf{p}_{\mathrm{BA}}$ :

(1-a) Compute the initial value of $\mathbf{p}_{\mathrm{BA} \text { (init) }}$ with GPS based global localization results of the two vehicles.

(1-b) In a certain error range around $\mathbf{p}_{\mathrm{BA}(\mathrm{init})}$, randomly generate an initial population of $\mathbf{p}_{\mathrm{BA}}$ i.e. $\left\{\mathbf{p}_{\mathrm{BA}(k)} \mid k=1,2, \ldots, n\right\}$. With an intention to examine the robustness of the method, we deliberately exaggerate this initial error range to be \pm 30 meters in position and \pm 30 degrees in orientation.

2. Evolution: iteratively perform the following sub-steps as follows:

(2-a) Compute the likelihood value (or fitness value in traditional genetic algorithm terms) of each individual in the population, according to (6).

(2-b) Compute mean likelihood value of the population. For an individual, if its likelihood value is above the mean likelihood value, assign the individual to the elite group; otherwise, assign it to the inferior group.

(2-c) Mutate the individuals in the elite group. For an individual, if its mutation has higher likelihood value than its own, then replace this individual with its mutation; otherwise, just keep this individual originally in the elite group.

$$
\begin{aligned}
& F O R \mathbf{p}_{\mathrm{BA}(k)} \in\left\{\mathbf{p}_{\mathrm{BA}(\text { elite })}\right\} \\
& \mathbf{p}_{\mathrm{BA}(k)} *=\operatorname{mutate}\left(\mathbf{p}_{\mathrm{BA}(k)}\right) \\
& I F F_{c}\left(\mathbf{p}_{\mathrm{BA}(k)} *\right)>F_{c}\left(\mathbf{p}_{\mathrm{BA}(k)}\right) T H E N \mathbf{p}_{\mathrm{BA}(k)}=\mathbf{p}_{\mathrm{BA}(k)} * \\
& E N D F O R
\end{aligned}
$$

Among the elite group, the best individual is an exception, which gets more times (for example, 100 times) of mutation. If no mutation is better, then just keep the best individual unchanged; otherwise, keep the best mutation to replace the original best individual.

(2-d) Replace the inferior group with new individuals; more specifically, replace each individual in the inferior group with a new individual that is generated from old individuals by applying the following genetic operations with specified probabilities:

$$
\text { (2-d-i) Copy the best individual (only performed once). }
$$

(2-d-ii) Randomly select an individual from the elite group and mutate it to be the new individual.

(2-d-iii) Randomly select two individuals from the elite group, create a new individual by executing crossover on them and mutating the crossover result. Two sorts of crossover are designed:

Crossover I: Mix the position parts and orientation parts of the two individuals. Let the two elite individuals be denoted as $\mathbf{p}_{\mathrm{BA}(\mathrm{e} 1)}=\left[x_{\mathrm{BA}(\mathrm{e} 1)}, y_{\mathrm{BA}(\mathrm{e} 1)}, \theta_{\mathrm{BA}(\mathrm{e} 1)}\right]^{\mathrm{T}}$ and $\mathbf{p}_{\mathrm{BA}(\mathrm{e} 2)}=\left[x_{\mathrm{BA}(\mathrm{e} 2),} y_{\mathrm{BA}(\mathrm{e} 2)}\right.$, $\left.\theta_{\mathrm{BA}(\mathrm{e} 2)}\right]^{\mathrm{T}}$; the new individual is generated as follows:

$$
\begin{aligned}
& \mathbf{p}_{\mathrm{BA}(\text { new })} \\
& =\left[x_{\mathrm{BA}(\mathrm{e} 1)}, y_{\mathrm{BA}(\mathrm{e} 1)}, \theta_{\mathrm{BA}(\mathrm{e} 2)}\right]^{T} \text { or }\left[x_{\mathrm{BA}(\mathrm{e} 2)}, y_{\mathrm{BA}(\mathrm{e} 2)}, \theta_{\mathrm{BA}(\mathrm{e} 1)}\right]^{T}
\end{aligned}
$$

Crossover II: Make a random linear combination of the two individuals (the $\lambda$ is a randomly generated real value in $[0,1]$ :

$$
\mathbf{p}_{\mathrm{BA}(\text { new })}=\lambda \mathbf{p}_{\mathrm{BA}(\mathrm{e} 1)}+(1-\lambda) \mathbf{p}_{\mathrm{BA}(\mathrm{e} 2)}
$$

(2-d-iv) Re-initialization: Create the new individual according to GPS based vehicle global localization results and the error range, as in the initialization process. This reinitialization practice is to keep the diversity of the population.

When two vehicles meet or re-meet, the initialization step is performed once and the sub-steps in evolution are repeatedly performed. A dynamic scheme of the genetic algorithm is used: the generation of $\mathbf{p}_{\mathrm{BA}}$ individuals from last period is propagated to the current period, according to the change of local map coordinates systems. As long as the vehicles are in the neighborhood and in cooperation, the evolution step can be performed unceasingly. As a result, we only need to assign few times of evolution for each period (for example, once), which largely reduces computational burden at one period; moreover, as the evolution continuous unceasingly, the dynamic scheme of genetic algorithm will finally converge to the optimum. In our tests, the genetic algorithm usually converges to the optimum in only few periods (no more than one second).

\section{Multi-Vehicle CoOperative Local Mapping AND MOVING OBJECT DETECTION}

The purpose of this section is not to propose certain moving object detection method, but to demonstrate a scheme of multivehicle cooperative local mapping and moving object detection, where the occupancy grid maps merging method can be applied to merge the moving object detection results of different vehicles. Here, we adopt the consistency based approach and the motion object map based approach [4] [5] for single vehicle moving object detection.

Consistency-based detection: given a new scan of range measurements and previously constructed occupancy grid maps, the idea is to find the inconsistent part between range measurements and free space in the local occupancy grid map. If a range point is detected on a location of previously free space, then it is regarded as a moving point. The range data are clustered into segments; for a segment, if the number of moving points is larger than a half of the total points, then the segment is identified as potential moving object. 
Moving object map based detection: a local moving object map is created to store information about previously detected moving objects; each cell in the moving object map stores a value indicating the number of observations that a moving object has been observed at that cell location. If the cell value is above certain threshold, the range point associated with this cell is regarded as a moving point.

During multi-vehicle cooperation, a vehicle (referred to as ego vehicle) will merge the local occupancy grid map of another vehicle into its own occupancy grid map, using the method introduced in Section 3. The detected moving objects of another vehicle can also be transformed into the ego vehicle reference and fused with the detected moving objects of the ego vehicle: if a detected moving object of another vehicle and a detected moving object of the ego vehicle have at least partial overlap, then the two objects are regarded as the same object and fused into one object.

\section{EXPERIMENT}

Two CyCab vehicle platforms (developed by IMARA team, INRIA) [16] are used for real data experiments which are carried out in INRIA campus: each vehicle is equipped with a RTK-GPS, odometer sensor, and an IBEO laser scanner. A RTK-GPS can achieve centimeter-level positioning accuracy; however, the RTK-GPS outputs are intentionally degraded with error noise in order to simulate vehicle systems with lowaccuracy GPS. Let the GPS output of one vehicle be degraded with an error bias component of $(8 \mathrm{~m}, 6 \mathrm{~m})$ and a noise component of mean 0 and standard deviation $7 \mathrm{~m}$; let the GPS output of the other vehicle be degraded with an error bias component of $(-9 \mathrm{~m}, 6 \mathrm{~m})$ and a noise component of mean 0 and standard deviation $8 \mathrm{~m}$. The degraded RTK-GPS outputs are used in the tests; the time of the two vehicle systems are related to the GPS universal time.

Each vehicle performs occupancy grid based local SLAM using the method in Section 2.1; the local SLAM outputs are used to correct odometer data. The degraded GPS measurements are fused with corrected odometer data to perform global localization, using the method in Section 2.2.

When the two vehicles are in cooperation mode-service detection for initiating cooperation is based on vehicle global localization result - cooperative local mapping and moving object detection are performed using the method introduced in Section 3 and Section 4. Some example results are demonstrated in Fig.2-4.

In Fig.2, the top two sub-figures are the local occupancy grid maps built by the two vehicles. The bottom-left sub-figure shows the merging effect using the low-accuracy GPS based localization results; there is large alignment inconsistency between the two local maps. The bottom-right sub-figure shows the occupancy grid maps merging effect using the method introduced in Section 3; the two local maps are aligned correctly.

Another example of occupancy grid maps merging is demonstrated in Fig.3, the top two sub-figures are two local occupancy grid maps. The bottom-left sub-figure shows the merging effect of map occupied cells; the bottom-right subfigure shows the merged occupancy grid map. As we can see, there is considerable maps inherent inconsistency between the two local maps; however, the proposed occupancy grid maps merging method is insensitive to the maps inherent inconsistency and merges the two local maps correctly.

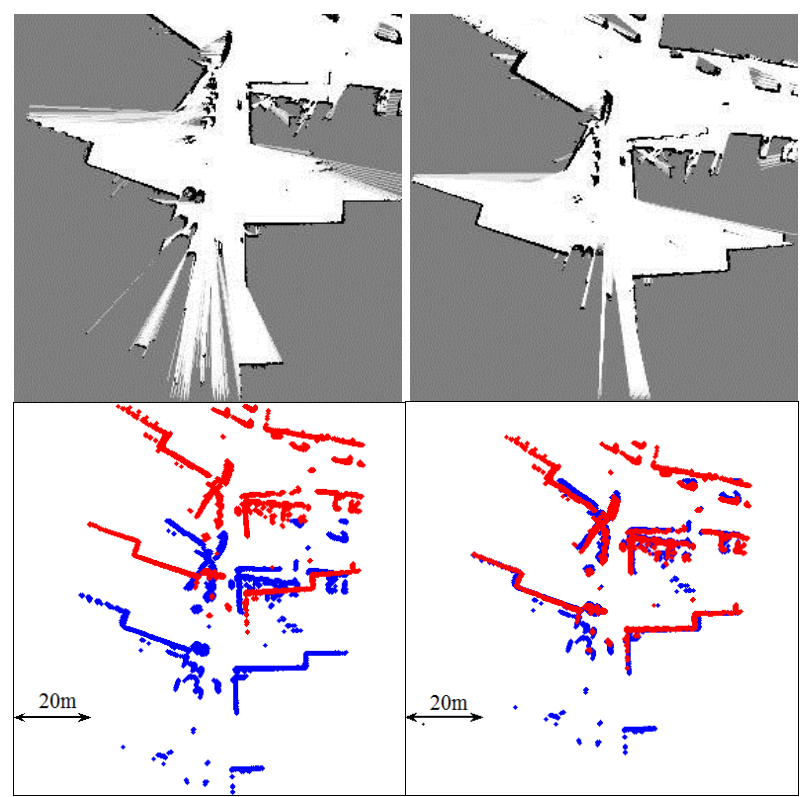

Fig 2 (top) local maps of two vehicles; (bottom-left) local maps merging using low-accuracy GPS localization results; (bottom-right) consistent local maps merging

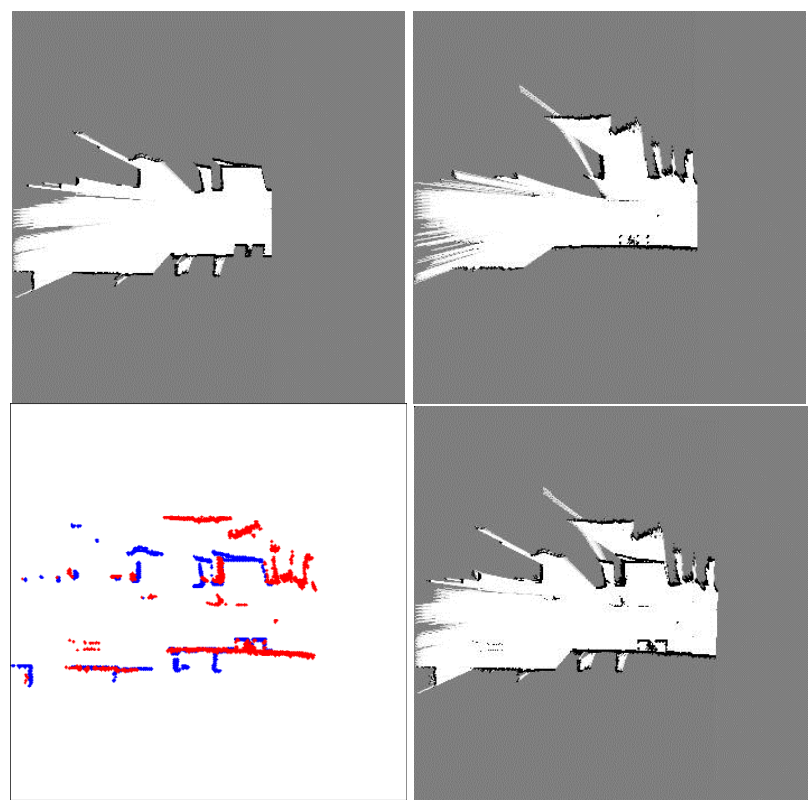

Fig 3 (top) local maps of two vehicles; (bottom-left) merged maps occupied cells; (bottom-right) merged occupancy grid map

Cooperative moving object detection is demonstrated in Fig.4, each of the left two sub-figures shows the local map and moving object detection result of one single vehicle; the 
detected moving objects are marked by blue boxes. The merged occupancy grid map and moving object detection result are shown in bottom-right sub-figure. Compared with the bottom-left sub-figure, the bottom-right sub-figure shows a more complete view for the vehicle.

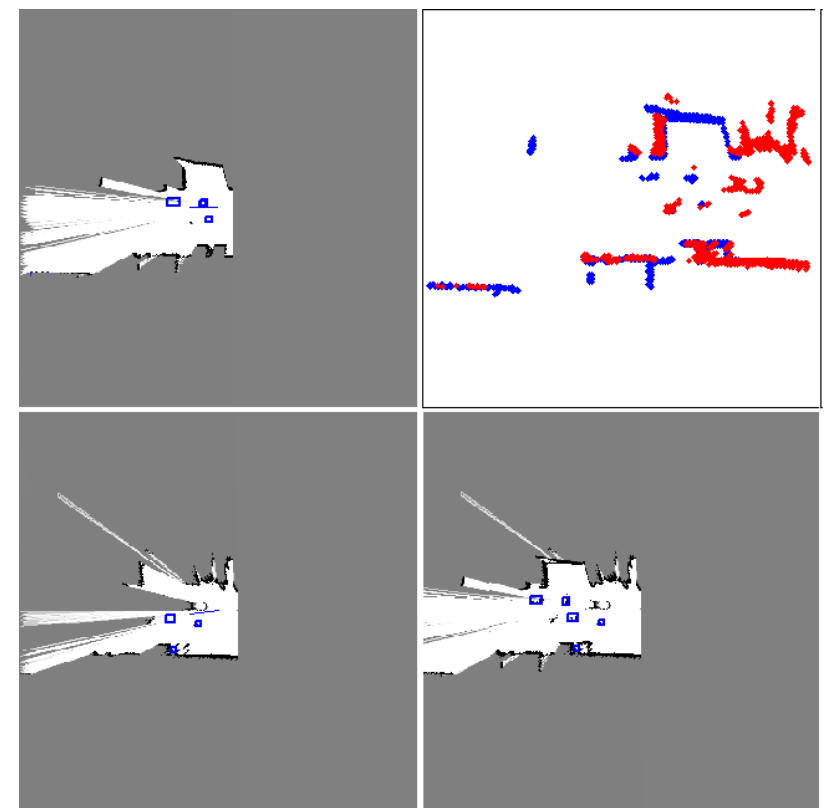

Fig 4 (left) local maps and single vehicle moving object detection; (top-right) local maps merging; (bottom-right) cooperative moving object detection

\section{CONCLUSION}

This paper proposes a new method for occupancy grid maps merging, which uses an objective function based on occupancy likelihood and uses genetic algorithm implemented in a dynamic scheme to optimize the objective function. The occupancy grid maps merging method is used for multi-vehicle cooperative local mapping and moving object detection. The introduced methods are tested on real-data experiments and several performance examples are given to demonstrate the effectiveness of the methods. Cooperative moving object detection can be valuable for many scenarios such as overtaking scenarios that are challenging for single vehicle system.

In future, more advanced moving object detection methods can be incorporated into the scheme of multi-vehicle cooperative local mapping and moving object detection. In the presented application of cooperative moving object detection, moving objects are detected based on local map of each single vehicle first and then merged. As merged local map can provide more complete view than the local map of each single vehicle, moving object detection based on merged local map might yield better detection result. This can also be a direction of future works.

\section{ACKNOWLEDGMENT}

This research work is supported by the French national project "ABV" (Automatisation Basse Vitesse).

\section{REFERENCE}

[1] S. Thrun, "Robotic mapping: A survey", Technical Report CMU-CS-02111, School of Computer Science, Carnegie Mellon University, 2002

[2] H. Durrant-Whyte, T. Bailey, "Simultaneous localization and mapping: part I", IEEE Robotics \& Automation Magazine, 13(2), 2006, pp.99-110

[3] T. Bailey, H. Durrant-Whyte, "Simultaneous localization and mapping (SLAM): part II”, IEEE Robotics \& Automation Magazine, 13(3), 2006, pp.108-117

[4] C.C. Wang, "Simultaneous Localization, Mapping and Moving Object Tracking", PhD Thesis, Robotics Institute, Carnegie Mellon University, Pittsburgh PA, 2004

[5] T.D. Vu, "Vehicle perception: Localization, mapping with detection, classification and tracking of moving objects", $\mathrm{PhD}$ Thesis, Institut National Polytechnique de Grenoble, 2009

[6] Y. Khaled, M. Tsukada, J. Santa, J. Choi, T. Ernst, "A usage oriented analysis of vehicular networks: from technologies to applications", Journal of Communications, 4(5), 2009, pp.357-368

[7] R. Madhavan, K. Fregene, L.E. Parker, "Distributed cooperative outdoor multirobot localization and mapping", Autonomous Robots 17, 2004, pp.23-39

[8] A. Howard, L.E. Parker, G.S. Sukhatme, "The SDR experience: Experiments with a large-scale heterogeneous mobile robot team", Experimental Robotics IX, STAR 21, Springer-Verlag Berlin Heidelberg, 2006, pp.121-130

[9] A. Howard, "Multi-robot simultaneous localization and mapping using particle filters", International Journal of Robotics Research, 25(12), 2006, pp.1243-1256

[10] L. Carlone, M.K. Ng, J. Du, B. Bona, M. Indri, "Simultaneous localization and mapping using rao-blackwellized particle filters in multi robot systems", Journal of Intelligent and Robotic Systems, 63(2), 2011, pp.283-307

[11] A. Birk, S. Carpin, "Merging occupancy grids from multiple robots", Proceedings of the IEEE, 94(7), 2006, pp.1384-1397

[12] A. Elfes, "Occupancy grids: a probabilistic framework for robot perception and navigation", PhD Thesis, Carnegie Mellon University, 1989

[13] S. Thrun, "Learning occupancy grids with forward sensor models", Autonomous Robots 15, 2003, pp.111-127

[14] G. Dedeoglu, G. Sukhatme, "Landmark-based matching algorithm for cooperative mapping by autonomous robots", International Symposium on Distributed Autonomous Robotics Systems, 2000, pp.251-260

[15] K.F. Man, K.S. Tang, S. Kwong, "Genetic algorithms", New York: Springer-Verlag, 1999

[16] http://www.science.gouv.fr/fr/actualites/bdd/res/3310/cycab-la-voitureintelligente-et-non-polluante/

[17] H. Li, F. Nashashibi, "Multi-vehicle cooperative localization using indirect vehicle-to-vehicle relative pose estimation", IEEE International Conference on Vehicular Electronics and Safety, 2012, pp.267-272 